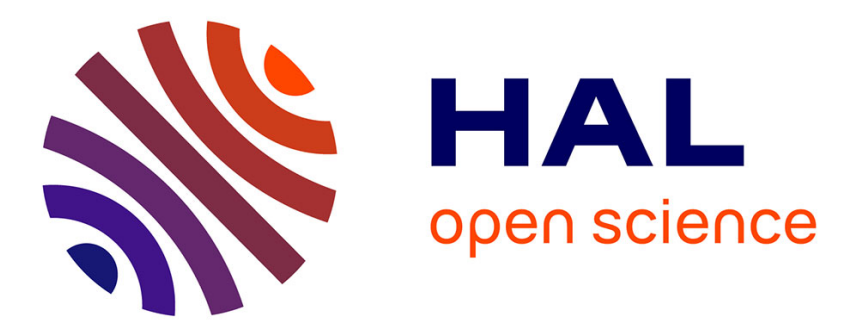

\title{
Anti-windup Design for Linear Discrete-time Systems Subject to Actuator Additive Faults and Saturations
}

Camille Sarotte, Julien Marzat, Hélène Piet-Lahanier, Marco Galeotta, Gérard Ordonneau

\section{- To cite this version:}

Camille Sarotte, Julien Marzat, Hélène Piet-Lahanier, Marco Galeotta, Gérard Ordonneau. Antiwindup Design for Linear Discrete-time Systems Subject to Actuator Additive Faults and Saturations. American Control Conference 2019, Jul 2019, PHILADELPHIE, United States. hal-02345444

\section{HAL Id: hal-02345444 \\ https://hal.science/hal-02345444}

Submitted on 4 Nov 2019

HAL is a multi-disciplinary open access archive for the deposit and dissemination of scientific research documents, whether they are published or not. The documents may come from teaching and research institutions in France or abroad, or from public or private research centers.
L'archive ouverte pluridisciplinaire HAL, est destinée au dépôt et à la diffusion de documents scientifiques de niveau recherche, publiés ou non, émanant des établissements d'enseignement et de recherche français ou étrangers, des laboratoires publics ou privés. 


\title{
Anti-windup Design for Linear Discrete-time Systems Subject to Actuator Additive Faults and Saturations
}

\author{
Camille Sarotte $^{1}$, Julien Marzat ${ }^{1}$, Hélène Piet-Lahanier ${ }^{1}$, Marco Galeotta ${ }^{2}$ and Gérard Ordonneau ${ }^{3}$
}

\begin{abstract}
In this paper a method is proposed to design an anti-windup scheme for discrete time linear systems with input saturations and actuator additive failures. This method provides a fault tolerant system reconfiguration mechanism with a control law which compensates for the estimated actuator additive faults and maintains the overall system stability in spite of actuator saturations. The design approach is derived from the solution of linear matrix inequalities (LMI) to guarantee the stability regions. For that purpose the fault tolerant control method is based on a linear quadratic regulator (LQR) and a fault estimator for compensation purposes. This method was tested in realistic simulations with the software Carins (CNES) on a pressure and mass flow rate model of a cryogenic test bench cooling circuit.
\end{abstract}

\section{INTRODUCTION}

Modern technological systems rely on sophisticated control systems to meet increased performance and safety requirements. A conventional control design for a complex system may result in an unsatisfactory performance, or even instability, in the event of malfunctions in actuators, sensors or other system components. The consequences could be damage to technical parts of the systems or to their environments. The need for increased system reliability leads to the development of health monitoring systems. In the case of actuator failures, it is necessary to be able to handle emergency situations that can affect a system performance. The failure should be detected quickly and if possible a control law should be designed to accommodate the presence of faults, for example see [1], [2]. The main objective of a Fault tolerant control system (FTCS) is to maintain, with a control reconfiguration mechanism, current performances close to the desirable ones and preserve stability conditions in the presence of component and/or instrument faults. But due to physical actuators characteristics or performances, unlimited control signals are not available and saturations should be taken into account in the control law design. Multiple solutions have been studied to compensate for a decrease in system performance caused by the saturation of one or more actuators, one way is to add a so-called antiwindup command, another way is to use direct synthesis methods by taking into account the saturations in the control law. Direct synthesis methods aims at taking into account the nonlinearities due to the saturations in the development of the control law in order to preserve the performances while improving the stability [3], [4]. Some methods determine

\footnotetext{
${ }^{1}$ DTIS, ONERA, Université Paris-Saclay, F-91123 Palaiseau, France \{firstname. lastname\} @onera.fr

${ }^{2}$ DLA, CNES, Paris, France

${ }^{3}$ DPE, ONERA, Université Paris-Saclay, F-91123 Palaiseau, France
}

a stabilizing gain based on a stochastic linearization of the saturations. The choice of this gain is very restrictive because it limits the stability domain. Indeed, these methods require to determine the parameters on which the gain depends [5], these parameters making it possible to ensure a semi-global stability. Methods that consist of a state, output or linear error feedback to remain below the limits of the actuator often have a slow dynamical behavior in order to avoid overshoots [3], [6], [7]. These methods are based on low gains whose values are limited to avoid saturation and therefore have a relatively slow response in time which is undesirable for fault tolerant control. The gain choice is then carried out by the resolution of linear matrix inequalities and a Riccatti equation whose solution depends on weighting matrices that must respect certain constraints in order to not exceed the limiting value. However, in these works, the choice of these weighting matrices on the stability domain is not clearly established. These methods have therefore to be improved thanks mainly to two types of methods: the addition of a control part based on high-gain methods or the addition of a nonlinear part to the command [8], [9], [10]. The use of these transient performance enhancement methods also requires the selection of parameters that can be constraining. These parameters make it possible to adjust the control in order to improve the performance of the closed loop of the system, in particular by activating the nonlinear part of the control law when one moves away from the reference to follow, in order to respect the limit on the system inputs. This type of methods remains close to the anti-windup. The idea of the anti-windup approach is to add a state, output or error feedback so that the actuator remains within its limits. This consists in neglecting the saturation in the first stage of the control design process, and then to add some problemspecific schemes to deal with the adverse effects caused by saturation. In the case of discrete systems, our interest is the development of control laws that provide a semi-global convergence on any arbitrarily large set of state space. They usually have a simpler structure and the controller is less sensitive to model and disturbance uncertainties. The system performance one wants to achieve can range from the classic system stabilization problem to expanding the area of attraction, rejecting disturbances, and regulating the output of the system [11]. The advantage of the presented control method is that it studies the determination of the stability regions of a discrete linear system over time and allows to determine an anti-windup control law which ensures the asymptotic stability of the saturated system. Unlike conventional antiwindup methods based on the resolution of bilinear matrix 
inequalities, this method is relatively simple and proposes an iterative algorithm of linear matrix inequalities in the same spirit as [12]. In this approach, the set of admissible initial states and its associated domain of stability are determined to take into account the compensation of additive actuator faults. The first part presented in Section 2 consists in the description of the considered system, then in Section 3 the design of an anti-windup control law is proposed in order to determine a set of admissible initial states and its associated domain of stability. This method has been validated in Section 4 on realistic simulations of a cryogenic combustion test bench cooling circuit.

\section{SYSTEM DESCRIPTION}

The system considered is:

$$
(\Sigma)\left\{\begin{array}{c}
X_{k+1}=A_{c} X_{k}+B_{c} U_{k}+B_{c} f_{k} \\
Y_{k+1}=C X_{k+1}
\end{array}\right.
$$

where $X_{k} \in \Re^{n}$ is the state vector, $Y_{k} \in \Re^{m}$ is the measured output, $U_{k} \in \Re^{l}$ is the known input, $f_{k} \in \Re^{l}$ is the unknown actuator failure, $A_{c} \in \Re^{n \times n}$ the state matrix, $B_{c} \in \Re^{n \times l}$ the known input distribution matrix and $C \in \Re^{m \times n}$ the output distribution matrix, with $m \leq n$. In the unsaturated case, we can use a control law of the form:

$$
U_{k}:=-B_{c}^{+} B_{c} \hat{f}_{k}+W_{c}\left(\hat{X}_{c, k}-\bar{X}_{k}\right)
$$

where we denote $B_{c}^{+}$as the pseudo-inverse of $B_{c},-B_{c}^{+} B_{c} \hat{f}_{k}$ is the fault compensation part and $W_{c}\left(\hat{X}_{c, k}-\bar{X}_{k}\right)$ is the reconfiguration part. The fault magnitude estimation $\hat{f}_{k}$ is assumed to be estimated with a filter and the gain $W_{c}$ is calculated with a linear quadratic regulator [13], [14]. The reference state trajectory $\bar{X}_{k}$ is predetermined and its dynamics is given by:

$$
\bar{X}_{k+1}=A_{c} \bar{X}_{k}+B_{c} \bar{U}_{k}
$$

with $\bar{U}_{k}$ the nominal input. Since the fault tolerant control is activated once a fault has been detected, the nominal input can be chosen as the mean input over a sliding window during nominal performances.

The control law can be alternatively written as

$$
U_{k}:=-B_{c}^{+} B_{c} \hat{f}_{k}+W_{c} e_{c, k}+W_{c} \eta_{k}
$$

The dynamics of the augmented state is expressed as:

$$
\zeta_{k+1}=\left[\begin{array}{cc}
A_{c}+B_{c} W_{c} & B_{c} W_{c} \\
0 & N_{c}
\end{array}\right] \zeta_{k}
$$

where $\zeta_{k}:=\left[\begin{array}{ll}\eta_{k} & e_{c, k}\end{array}\right]^{T}$, with $e_{c, k}=\hat{X}_{c, k}-X_{k}$ the estimation error, $\eta_{k}=X_{k}-\bar{X}_{k}$ the reconfiguration error and $\bar{X}_{k}$ the state reference. $N_{c}$ is the gain of an observer ensuring the estimation error convergence so that its dynamics reduces to $e_{c, k+1}=N_{c} e_{c, k}$.

For the nominal system, the gain $W_{c}$ must stabilize $\left(A_{c}+\right.$ $\left.B_{c} W_{c}\right)$. Since the pair $\left(A_{c}, B_{c}\right)$ is assumed to be controllable, a Linear-Quadratic Regulator (LQR) formulation can be adopted where $W_{c}$ is selected to minimize

$$
J_{k}:=\sum_{k} X_{k}^{T} Q_{k} X_{k}+U_{k}^{T} R_{k} U_{k}
$$

where $Q_{k}$ and $R_{k}$ are symmetric positive definite design matrices.

When the input is assumed to be saturated the system considered becomes:

$$
\left\{\begin{array}{c}
X_{k+1}=A_{c} X_{k}+B_{c} \operatorname{sat}\left(U_{k}\right)+B_{c} f_{k} \\
Y_{k+1}=C X_{k+1}
\end{array}\right.
$$

$$
\text { with } \operatorname{sat}\left(U_{k}\right):=\left\{\begin{array}{cc}
U_{i, s a t} & \text { if } U_{i, k}>U_{i, s a t} \\
U_{i, k} & \text { if }-U_{i, s a t} \leq U_{i, k} \leq U_{i, s a t} \\
-U_{i, s a t} & \text { if } U_{i, k}<-U_{i, s a t}
\end{array}\right\}
$$

where $U_{k} \in \Re^{l}$ is the control law and $U_{\text {sat }} \in \Re^{l^{+}}$is the actuator limit, $\forall i=1, \ldots, l$.

\section{DESIGN OF THE ANTI-WINDUP CONTROL LAW}

The aim of this section is to design an anti-windup control law in order to ensure the asymptotic stability of the system with a saturated input for a given set of initial conditions and determine the associated stability domain.

We want to determine the anti-windup gain matrix $E_{c}$ such that for a set $\mathcal{S}$ of admissible initial states $\left(\zeta_{0} \in \mathcal{S}\right)$, the corresponding trajectory converges asymptotically to the origin of the subset $\mathcal{E} \subset \mathcal{S}$. Then, $\mathcal{E}$ is a region of asymptotic stability. For that, we want to determine a new control law of the form $U_{k}{ }^{+}=U_{k}-G \zeta_{k}$ when the control law $U_{k}$ reaches its bounds with $G \in \Re^{l \times 2 n}$.

The reference state dynamics for the anti-windup strategy is given as:

$$
\begin{aligned}
\bar{X}_{k+1} & :=A_{c} \bar{X}_{k}+B_{c} \bar{U}_{k}+E_{c}\left(\operatorname{sat}\left(U_{k}\right)-U_{k}\right) \\
U_{k} & :=\bar{U}_{k}-B_{c}^{+} B_{c} \hat{f}_{k}+W_{c}\left(\hat{X}_{c, k}-\bar{X}_{k}\right)
\end{aligned}
$$

If the control law is saturated then $U_{k}= \pm U_{\text {sat }}$ :

$$
\begin{aligned}
\bar{X}_{k+1} & =A_{c} \bar{X}_{k}+B_{c} \bar{U}_{k} \\
& +E_{c}\left( \pm U_{s a t}-\bar{U}_{k}+B_{c}^{+} B_{c} \hat{f}_{k}-W_{c} e_{c, k}-W_{c} \eta_{k}\right)
\end{aligned}
$$

We can then write:

$$
\begin{aligned}
X_{k+1} & -\bar{X}_{k+1}=A_{c}\left(X_{k}-\bar{X}_{k}\right)+B_{c} \bar{U}_{k}+B_{c} f_{k} \\
& -B_{c} \hat{f}_{k}-B_{c} \bar{U}_{k}+B_{c} W_{c} e_{c, k}+B_{c} W_{c} \eta_{k} \\
& +E_{c}\left( \pm U_{s a t}-\bar{U}_{k}+B_{c}^{+} B_{c} \hat{f}_{k}-W_{c} e_{c, k}-W_{c} \eta_{k}\right)
\end{aligned}
$$

which gives

$$
\begin{aligned}
\eta_{k+1} & =A_{c} \eta_{k}+B_{c} W_{c} e_{c, k}+B_{c} W_{c} \eta_{k} \\
& +E_{c}\left( \pm U_{s a t}-\bar{U}_{k}+B_{c}^{+} B_{c} \hat{f}_{k}-W_{c} e_{c, k}-W_{c} \eta_{k}\right)
\end{aligned}
$$

we then have:

$$
\zeta_{k+1}=\left[\begin{array}{cc}
A_{c}+B_{c} W_{c} & B_{c} W_{c} \\
0 & N_{c}
\end{array}\right] \zeta_{k}-\left(\mathcal{R} E_{c}\right) \Psi\left(\mathcal{K} \zeta_{k}\right)
$$


with

$$
\Psi(u):=\left\{\begin{array}{c}
u_{i}-U_{i, s a t}+\bar{U}_{i, k}-\left(B_{c}^{+} B_{c} \hat{f}_{k}\right)_{i} \\
\text { if } u_{i}+\bar{U}_{i, k}-\left(B_{c}^{+} B_{c} \hat{f}_{k}\right)_{i}>U_{i, s a t} \\
0 \\
\text { if }-U_{i, s a t} \leq u_{i}+\bar{U}_{i, k}-\left(B_{c}^{+} B_{c} \hat{f}_{k}\right)_{i} \leq U_{i, s a t} \\
u_{i}+U_{i, s a t}+\bar{U}_{i, k}-\left(B_{c}^{+} B_{c} \hat{f}_{k}\right)_{i} \\
\text { if } u_{i}+\bar{U}_{i, k}-\left(B_{c}^{+} B_{c} \hat{f}_{k}\right)_{i}<-U_{i, s a t}
\end{array}\right.
$$

where $\mathcal{R}=\left[\begin{array}{c}I_{n} \\ 0\end{array}\right], \mathcal{K}=\left[\begin{array}{ll}W_{c} & W_{c}\end{array}\right], \forall i=1, \ldots, l$.

The set of admissible initial states $\mathcal{S}$ considered will be defined as a polyhedral set and the domain of stability $\mathcal{E}$ will be designed as an ellipsoid.

\section{A. Determination of the set of admissible initial states}

Lemma 1: Consider a matrix $G \in \Re^{l \times 2 n}$ and define the following polyhedral set:

$\mathcal{S}=\left[\zeta_{k} \in \Re^{2 n} ;-U_{i, s a t} \leq\left((\mathcal{K}-G) \zeta_{k}\right)_{i}+\bar{U}_{i, k}-\left(B_{c}^{+} B_{c} \hat{f}_{k}\right)_{i}\right.$ $\left.\leq U_{i, s a t} ; \forall i=1, \ldots, l\right]$

For the function $\Psi(u)$ defined in (13), if $\zeta_{k} \in \mathcal{S}$ then:

$$
\Psi\left(\mathcal{K} \zeta_{k}\right)^{T} T\left[\Psi\left(\mathcal{K} \zeta_{k}\right)-G \zeta_{k}\right] \leq 0
$$

for any matrix $T \in \Re^{l \times l}$ diagonal and positive definite.

This property will be used in the proof of Theorem 2 (34) to find the gain $E_{c}$ depending on the choice of $G$ to ensure the exponential asymptotic stability of the system.

Proof:

(1) We consider the case where:

$$
\left(\mathcal{K} \zeta_{k}\right)_{i}+\bar{U}_{i, k}-\left(B_{c}^{+} B_{c} \hat{f}_{k}\right)_{i}>U_{i, s a t}
$$

then,

$$
\left(\mathcal{K} \zeta_{k}\right)_{i}-U_{i, s a t}+\bar{U}_{i, k}-\left(B_{c}^{+} B_{c} \hat{f}_{k}\right)_{i}>0
$$

We have:

$$
\Psi\left(\mathcal{K} \zeta_{k}\right)=\left(\mathcal{K} \zeta_{k}\right)_{i}-U_{i, s a t}+\bar{U}_{i, k}-\left(B_{c}^{+} B_{c} \hat{f}_{k}\right)_{i}
$$

If $\zeta_{k} \in \mathcal{S},\left((\mathcal{K}-G) \zeta_{k}\right)_{i}+\bar{U}_{i, k}-\left(B_{c}^{+} B_{c} \hat{f}_{k}\right)_{i} \leq U_{i, s a t}$, then:

$$
\begin{aligned}
& {\left[\left(\mathcal{K} \zeta_{k}\right)_{i}-U_{i, s a t}+\bar{U}_{i, k}-\left(B_{c}^{+} B_{c} \hat{f}_{k}\right)_{i}\right]^{T}} \\
& T_{i, i}\left[\left((\mathcal{K}-G) \zeta_{k}\right)_{i}-U_{i, s a t}+\bar{U}_{i, k}-\left(B_{c}^{+} B_{c} \hat{f}_{k}\right)_{i}\right] \leq 0
\end{aligned}
$$

for $T$ diagonal and positive definite.

(2) We consider the case where:

$$
\left(\mathcal{K} \zeta_{k}\right)_{i}+\bar{U}_{i, k}-\left(B_{c}^{+} B_{c} \hat{f}_{k}\right)_{i}<U_{i, s a t}
$$

then,

$$
\left(\mathcal{K} \zeta_{k}\right)_{i}+U_{i, s a t}+\bar{U}_{i, k}-\left(B_{c}^{+} B_{c} \hat{f}_{k}\right)_{i}<0
$$

We have:

$$
\Psi\left(\mathcal{K} \zeta_{k}\right)=\left(\mathcal{K} \zeta_{k}\right)_{i}+U_{i, s a t}+\bar{U}_{i, k}-\left(B_{c}^{+} B_{c} \hat{f}_{k}\right)_{i}
$$

If $\zeta_{k} \in \mathcal{S},\left((\mathcal{K}-G) \zeta_{k}\right)_{i}+\bar{U}_{i, k}-\left(B_{c}^{+} B_{c} \hat{f}_{k}\right)_{i} \geq-U_{i, s a t}$, then:

$$
\begin{aligned}
& {\left[\left(\mathcal{K} \zeta_{k}\right)_{i}+U_{i, s a t}+\bar{U}_{i, k}-\left(B_{c}^{+} B_{c} \hat{f}_{k}\right)_{i}\right]^{T}} \\
& T_{i, i}\left[\left((\mathcal{K}-G) \zeta_{k}\right)_{i}+U_{i, s a t}+\bar{U}_{i, k}-\left(B_{c}^{+} B_{c} \hat{f}_{k}\right)_{i}\right] \leq 0
\end{aligned}
$$

for $T$ diagonal and positive definite.

(3) $\Psi\left(\mathcal{K} \zeta_{k}\right)=0$, then:

$$
\Psi\left(\mathcal{K} \zeta_{k}\right)^{T} T\left[\Psi\left(\mathcal{K} \zeta_{k}\right)-G \zeta_{k}\right]=0
$$

for $T$ diagonal and positive definite.

Theorem 1:

Define $\mathcal{E}(P)=\left[\zeta_{k} \in \Re^{2 n}, \forall i=1, \ldots, l ; \zeta_{k}{ }^{T} P \zeta_{k} \leq\right.$ $\left.1+\frac{\left(\left(B_{c}^{+} B_{c} \hat{f}_{k}\right)_{i}-\bar{U}_{i, k}\right)^{2}}{\left\|\left(B_{c}^{+} B_{c} \hat{f}_{k}\right)_{i}-\bar{U}_{i, k}\right\|^{2}}\right]$ with $P \in \Re^{2 n \times 2 n}$ a positive definite matrix and $\mathcal{W}:=P^{-1}$. If $\mathcal{W}$ satisfies (23) for each input value then $\mathcal{E}(P) \subset \mathcal{S}$.

$$
\left[\begin{array}{cc}
{\left[\begin{array}{cc}
\mathcal{W} & 0_{2 n, 1} \\
0_{1,2 n} & -1
\end{array}\right]} & \frac{\mathcal{W} \mathcal{K}_{i}^{T}-(G \mathcal{W})_{i}{ }^{T}}{\left\|\left(B_{c}^{+} B_{c} \hat{f}_{k}\right)_{i}-\bar{U}_{i, k}\right\|} \\
\frac{\mathcal{K}_{i} \mathcal{W}-(G \mathcal{W})_{i}}{\left\|\left(B_{c}^{+} B_{c} \hat{f}_{k}\right)_{i}-\bar{U}_{i, k}\right\|} & \frac{U_{i, s a t}{ }^{2}}{\left\|\left(B_{c}^{+} B_{c} \hat{f}_{k}\right)_{i}-\bar{U}_{i, k}\right\|^{2}}
\end{array}\right] \geq 0
$$

$\forall i=1, \ldots, l$

Assume that $\left(B_{c}^{+} B_{c} \hat{f}_{k}\right)_{i}-\bar{U}_{i, k} \neq 0$ since the case where the fault amplitude is equal to the reference input is not considered as a faulty case.

Proof:

By Schur's complement, (23) gives $\forall i=1, \ldots, l$ :

$$
\begin{aligned}
& {\left[\begin{array}{cc}
\mathcal{W} & 0_{2 n, 1} \\
0_{1,2 n} & -1
\end{array}\right]-\left[\begin{array}{c}
\frac{\mathcal{W} \mathcal{K}_{i}^{T}-(G \mathcal{W})_{i}{ }^{T}}{\left\|\mathcal{Y}_{i}\right\|} \\
1
\end{array}\right] \frac{U_{i, s a t}{ }^{-2}}{\left\|\mathcal{Y}_{i}\right\|^{-2}}} \\
& {\left[\begin{array}{cc}
\frac{\mathcal{K}_{i} \mathcal{W}-(G \mathcal{W})_{i}}{\left\|\mathcal{Y}_{i}\right\|} & 1
\end{array}\right] \geq 0}
\end{aligned}
$$

with $P=\mathcal{W}^{-1}, \mathcal{Y}_{i}=\left(B_{c}^{+} B_{c} \hat{f}_{k}\right)_{i}-\bar{U}_{i, k}, \mathcal{K}_{i}$ and $G_{i}$ are the $i^{\text {th }}$ lines of $\mathcal{K}$ and $G$. Then we have:

$$
\begin{aligned}
& {\left[\begin{array}{cc}
P & 0_{2 n, 1} \\
0_{1,2 n} & -1
\end{array}\right]-\left[\begin{array}{c}
\frac{\mathcal{K}_{i}^{T}-G_{i}^{T}}{\left\|\mathcal{Y}_{i}\right\|} \\
-1
\end{array}\right] \frac{U_{i, s a t}{ }^{-2}}{\left\|\mathcal{Y}_{i}\right\|^{-2}}\left[\frac{\mathcal{K}_{i}-G_{i}}{\left\|\mathcal{Y}_{i}\right\|}-1\right]} \\
& \geq 0
\end{aligned}
$$

Left multiplying by $\left[\begin{array}{c}\zeta_{k} \\ \frac{\mathcal{Y}_{i}}{\left\|\mathcal{Y}_{i}\right\|}\end{array}\right]^{T}$ and right multiplying by $\left[\begin{array}{c}\zeta_{k} \\ \frac{\mathcal{Y}_{i}}{\left\|\mathcal{Y}_{i}\right\|}\end{array}\right]$ we obtain:

$\left[\begin{array}{c}\zeta_{k} \\ \frac{\mathcal{Y}_{i}}{\left\|\mathcal{Y}_{i}\right\|}\end{array}\right]^{T}\left[\begin{array}{cc}P & 0_{2 n, 1} \\ 0_{1,2 n} & -1\end{array}\right]\left[\begin{array}{c}\zeta_{k} \\ \frac{\mathcal{Y}_{i}}{\left\|\mathcal{Y}_{i}\right\|}\end{array}\right] \geq$

$\left[\begin{array}{c}\zeta_{k} \\ \frac{\mathcal{Y}_{i}}{\left\|\mathcal{Y}_{i}\right\|}\end{array}\right]^{T}\left[\begin{array}{c}\frac{\mathcal{K}_{i}^{T}-G_{i}^{T}}{\left\|\mathcal{Y}_{i}\right\|} \\ -1\end{array}\right] \frac{U_{i, s a t}{ }^{-2}}{\left\|\mathcal{Y}_{i}\right\|^{-2}}\left[\frac{\mathcal{K}_{i}-G_{i}}{\left\|\mathcal{Y}_{i}\right\|}-1\right]\left[\begin{array}{c}\zeta_{k} \\ \frac{\mathcal{Y}_{i}}{\left\|\mathcal{Y}_{i}\right\|}\end{array}\right]$ then

$$
\begin{aligned}
& \zeta_{k}^{T} P \zeta_{k}-\frac{\mathcal{Y}_{i}^{2}}{\left\|\mathcal{Y}_{i}\right\|^{2}} \geq\left[\zeta_{k}^{T} \frac{\mathcal{K}_{i}^{T}-G_{i}^{T}}{\left\|\mathcal{Y}_{i}\right\|}-\frac{\mathcal{Y}_{i}}{\left\|\mathcal{Y}_{i}\right\|}\right] \\
& \frac{U_{i, s a t}{ }^{-2}}{\left\|\mathcal{Y}_{i}\right\|^{-2}}\left[\frac{\mathcal{K}_{i}-G_{i}}{\left\|\mathcal{Y}_{i}\right\|^{\prime}} \zeta_{k}-\frac{\mathcal{Y}_{i}}{\left\|\mathcal{Y}_{i}\right\|}\right]
\end{aligned}
$$


So $\zeta_{k} \in \mathcal{S}$ since $\zeta_{k}^{T} P \zeta_{k}-\frac{\mathcal{Y}_{i}^{2}}{\left\|\mathcal{Y}_{i}\right\|^{2}} \leq 1$ :

$$
\begin{gathered}
\left(\zeta_{k}^{T} P \zeta_{k}-\frac{\mathcal{Y}_{i}^{2}}{\left\|\mathcal{Y}_{i}\right\|^{2}}\right) \frac{U_{i, s a t}{ }^{2}}{\left\|\mathcal{Y}_{i}\right\|^{2}} \geq \\
{\left[\zeta_{k}^{T} \frac{\mathcal{K}_{i}^{T}-G_{i}^{T}}{\left\|\mathcal{Y}_{i}\right\|}-\frac{\mathcal{Y}_{i}}{\left\|\mathcal{Y}_{i}\right\|}\right]\left[\frac{K_{i}-G_{i}}{\left\|\mathcal{Y}_{i}\right\|} \zeta_{k}-\frac{\mathcal{Y}_{i}}{\left\|\mathcal{Y}_{i}\right\|}\right]} \\
\frac{U_{i, s a t}{ }^{2}}{\left\|\mathcal{Y}_{i}\right\|^{2}} \geq\left[\zeta_{k}{ }^{T} \frac{\mathcal{K}_{i}^{T}-G_{i}^{T}}{\left\|\mathcal{Y}_{i}\right\|}-\frac{\mathcal{Y}_{i}}{\left\|\mathcal{Y}_{i}\right\|}\right]\left[\frac{\mathcal{K}_{i}-G_{i}}{\left\|\mathcal{Y}_{i}\right\|} \zeta_{k}-\frac{\mathcal{Y}_{i}}{\left\|\mathcal{Y}_{i}\right\|}\right]
\end{gathered}
$$

we then have:

$-U_{i, s a t} \leq\left(\left(\mathcal{K}_{i}-G_{i}\right) \zeta_{k}+\bar{U}_{i, k}-\left(B_{c}^{+} B_{c} \hat{f}_{k}\right)_{i}\right) \leq U_{i, s a t}$

so that $\mathcal{E}(P) \subset \mathcal{S}$

\section{B. Determination of the associated domain of stability}

In this part, we denote: $\mathcal{A}:=\left[\begin{array}{cc}A_{c}+B_{c} W_{c} & B_{c} W_{c} \\ 0 & N_{c}\end{array}\right]$. $Z \in \Re^{n \times l}$ and $\Delta \in \Re^{l \times l}$ a diagonal positive definite matrix are parameters which will be chosen in order to maximize the size of the set of admissible initial states and ensure the exponential asymptotic stability of the augmented system (12).

\section{Theorem 2:}

The ellipse $\mathcal{E}(P)=\left[\zeta_{k} \in \Re^{2 n}, \forall i=1, \ldots, l ; \zeta_{k}{ }^{T} P \zeta_{k} \leq 1+\right.$ $\left.\frac{\left(\left(B_{c}^{+} B_{c} \hat{f}_{k}\right)_{i}-\bar{U}_{i, k}\right)^{2}}{\left\|\left(B_{c}^{+} B_{c} \hat{f}_{k}\right)_{i}-\bar{U}_{i, k}\right\|^{2}}\right]$ with $P=\mathcal{W}^{-1}$ is a region of exponential asymptotic stability for the augmented system, if for $E_{c}=$ $Z \Delta^{-1}$ :

$$
\left[\begin{array}{ccc}
\mathcal{W} & -(G \mathcal{W})^{T} & -\mathcal{W} \mathcal{A}^{T} \\
-(G \mathcal{W}) & 2 \Delta & Z^{T} \mathcal{R}^{T} \\
-\mathcal{A W} & \mathcal{R} Z & \mathcal{W}
\end{array}\right]>0
$$

for the considered Lyapunov candidate quadratic function:

$$
V\left(\zeta_{k}\right):=\zeta_{k}^{T} P \zeta_{k}, P=P^{T}>0, P \in \Re^{2 n \times 2 n}
$$

$V\left(\zeta_{k}\right)$ is a Lyapunov function since:

1) $\Delta V\left(\zeta_{k}\right)<0, \forall \zeta_{k} \in \mathcal{E}(P), \zeta_{k} \neq 0$

2) $\exists \alpha \in \Re^{+}, \Delta V\left(\zeta_{k}\right) \leq-\alpha V\left(\zeta_{k}\right)$

Proof: We calculate $\delta V\left(\zeta_{k}\right)$ :

$$
\begin{aligned}
\delta V\left(\zeta_{k}\right) & =V\left(\zeta_{k+1}\right)-V\left(\zeta_{k}\right) \\
& =\zeta_{k}{ }^{T} \mathcal{A}^{T} P \mathcal{A} \zeta_{k}-2 \zeta_{k}{ }^{T} \mathcal{A}^{T} P\left(\mathcal{R} E_{c}\right) \Psi(\mathcal{K} \zeta) \\
& +\Psi\left(\mathcal{K} \zeta_{k}\right)^{T}\left(\mathcal{R} E_{c}\right)^{T} P\left(\mathcal{R} E_{c}\right) \Psi\left(\mathcal{K} \zeta_{k}\right)-\zeta_{k}{ }^{T} P \zeta_{k}
\end{aligned}
$$

Using Lemma 1, we have:

$$
\begin{aligned}
\delta V\left(\zeta_{k}\right) & \leq-\left(\zeta_{k}^{T} \mathcal{A}^{T} P \mathcal{A} \zeta_{k}+2 \zeta_{k}^{T} \mathcal{A}^{T} P\left(\mathcal{R} E_{c}\right) \Psi\left(\mathcal{K} \zeta_{k}\right)\right. \\
& \left.-\Psi\left(\mathcal{K} \zeta_{k}\right)^{T}\left(\mathcal{R} E_{c}\right)^{T} P\left(\mathcal{R} E_{c}\right) \Psi\left(\mathcal{K} \zeta_{k}\right)+\zeta_{k}^{T} P \zeta_{k}\right) \\
& -2 \Psi\left(\mathcal{K} \zeta_{k}\right)^{T} T\left[\Psi\left(\mathcal{K} \zeta_{k}\right)-G \zeta_{k}\right]
\end{aligned}
$$

We can write this inequality under the form:

$$
\delta V\left(\zeta_{k}\right) \leq-\left[\begin{array}{ll}
\zeta_{k}^{T} & \Psi^{T}
\end{array}\right]\left[\begin{array}{cc}
X_{1} & X_{2} \\
X_{2}{ }^{T} & X_{3}
\end{array}\right]\left[\begin{array}{c}
\zeta \\
\Psi
\end{array}\right]
$$

With $X_{1}:=P-\mathcal{A}^{T} P \mathcal{A}, X_{2}:=\mathcal{A}^{T} P\left(\mathcal{R} E_{c}\right)-G^{T} T$, $X_{3}:=2 T-\left(\mathcal{R} E_{c}\right)^{T} P\left(\mathcal{R} E_{c}\right)$.
By Schur's complement, (31) gives:

$$
\left[\begin{array}{cc}
\mathcal{W} & -(G \mathcal{W})^{T} \\
-(G \mathcal{W}) & 2 \Delta
\end{array}\right]-\left[\begin{array}{c}
-\mathcal{W} \mathcal{A}^{T} \\
Z^{T} \mathcal{R}^{T}
\end{array}\right] P[-\mathcal{A} \mathcal{W}
$$

By multiplying from the left and from the right by

$$
\begin{gathered}
{\left[\begin{array}{cc}
P & 0 \\
0 & T
\end{array}\right] \text {, with } T:=\Delta^{-1} \text { et } P:=W^{-1} \text { we have: }} \\
{\left[\begin{array}{cc}
X_{1} & X_{2} \\
X_{2}{ }^{T} & X_{3}
\end{array}\right]>0}
\end{gathered}
$$

Then we have $\delta V\left(\zeta_{k}\right)<0$ for all $\zeta_{k} \in \mathcal{E}(P), \zeta_{k} \neq 0$, so $V\left(\zeta_{k}\right)$ is strictly decreasing along the system trajectories. Then $\mathcal{E}(P)$ is a stability region for the system. We can see that there always exists a positive scalar $\delta$ such that:

$$
\begin{aligned}
\delta V\left(\zeta_{k}\right) & \leq-\delta\left\|\zeta_{k}\right\|^{2}-\delta\|\Psi\|^{2} \leq-\delta\left\|\zeta_{k}\right\|^{2} \\
& \leq-\tilde{\delta} \zeta_{k}{ }^{T} P \zeta_{k}
\end{aligned}
$$

which ensures the exponential convergence with $\tilde{\delta}:=$ $\frac{\delta}{\lambda_{\max }(P)}$ and $\lambda_{\max }(P)$ the maximum eigenvalue of $P$.

\section{Application}

A control reconfiguration mechanism under actuator saturations for the cooling circuit of a cryogenic combustion bench, Mascotte (CNES/ONERA, [15]), is studied in this part. This bench has been developed to study heat transfers in the combustion chamber and jet separation in nozzles in the same conditions as for Vulcain 2 engine. The method proposed here consists in the design of a controller based on an unknown input observer by considering the fault to be the unknown input similar to [2] and the design of an anti-windup strategy in order to ensure the asymptotic stability of the saturated system for a given set of initial conditions and determine the stability domain. This FTC strategy permits to compensate the fault and maintain current performances in the presence of actuator saturations but also to converge if necessary to another reference state. The results are obtained with off-line tests based on real experimental data and the reconfiguration control law was validated on realistic simulations based on the established model. The system studied here is the water cooling system of Mascotte. This part of the system cools the combustion chamber. The detection of a leak or an obstruction in this part is a critical safety task for the bench operation. The water cooling circuit consists in different pipes sections with multiple pressure release valves and a tank at the inlet. The available measurements are pressure, mass flow and temperature. Sections are separated by sliding valves with additional pressure measurements. The model, the estimation and fault detection part has been presented in [14]. The system is regulated with a pressure dome-loaded regulator. The actuator is saturated since the pressure is limited by thermo-mechanical constraints. As in the previous work, the control loop operates once the fault has been detected during the steady state of the bench operation. 


\section{A. Model of the cooling circuit}

In this section we denote $\dot{m}$ the mass flow rate $(\mathrm{kg} / \mathrm{s})$, $\rho$ the density $\left(\mathrm{kg} / \mathrm{m}^{3}\right), S$ the surface $\left(\mathrm{m}^{2}\right), c$ the velocity of sound $(\mathrm{m} / \mathrm{s}), P$ the pressure $(\mathrm{Pa}), D$ the orifice diameter (m), $D_{h}$ the hydraulic diameter (m), $L$ the length (m), $\mu$ the dynamic viscosity (Pa.s) and $V$ the volume $\left(\mathrm{m}^{3}\right)$. The flow is assumed to stay monophasic, to be ideal (no force due to viscosity acts) and incompressible following Euler equations. The cavity section is assumed constant and the velocity of sound is defined as for an isentropic reaction in the orifice. We assume that the fluid flow velocity is small in comparison to the velocity of sound. The flow crossing cavities respects the mass balance equation, after integrating this equation over the cavity volume, we obtain:

$$
\frac{\partial P}{\partial t}=\frac{c^{2}}{V}\left(\dot{m}_{e}-\dot{m}_{s}\right)
$$

The flow crossing the orifice between the two cavities respects the momentum balance equation with friction forces, expressed with the Darcy-Weisbach and Blasius equations for moderate turbulent flows in a smooth pipe. After integrating this equation along the orifice volume we obtain:

$$
\frac{1}{S^{2}} \frac{\partial \dot{m}}{\partial t}+\frac{\Delta P}{V}=-0.316\left(\frac{4 \dot{m}}{\pi D \mu}\right)^{-\frac{1}{4}} \frac{L}{D_{h}} \frac{\dot{m}^{2}}{2 \rho V S^{2}}
$$

The model of this part of the cooling circuit is then:

$$
\begin{gathered}
\left\{\begin{array}{c}
\frac{\partial \dot{m}_{2 e}}{\partial t}=\theta_{1} \dot{m}_{2 e}^{\frac{7}{4}}-\theta_{2} \Delta P \\
\frac{\partial P_{2}}{\partial t}=-\theta_{3} \Delta \dot{m}
\end{array}\right. \\
\theta_{1}:=-0.316\left(\frac{4}{\pi D \mu}\right)^{-\frac{1}{4}} \frac{L}{D_{h}} \frac{1}{2 \rho V}, \quad \theta_{2}:=\frac{S^{2}}{V}, \quad \theta_{3}:=\frac{c^{2}}{V}
\end{gathered}
$$

The system (41) is linearized around a steady state trajectory and can be transformed into an equivalent discrete-time state space system with an Euler explicit scheme:

$$
\begin{gathered}
\left\{\begin{array}{c}
X_{k+1}=A_{k}(\bar{X}) X_{k}+B U_{k}+E D_{k} \\
Y_{k+1}=C X_{k+1}
\end{array}\right. \\
X:=\left[\begin{array}{cc}
\dot{m}_{2 e} & P_{2}
\end{array}\right]^{T}, \quad Y:=P_{2}, \quad U:=P_{1}, \quad D:=\dot{m}_{2 s}
\end{gathered}
$$

where $\dot{m}_{2 e}$ is the cavity 2 input mass flow rate, $P_{2}$ is the cavity 2 pressure, $P_{1}$ is the cavity 1 pressure and $\dot{m}_{2 s}$ is the cavity 2 unknown output mass flow rate. With $A_{k}$ the state matrix, $B$ the known input distribution matrix, $E$ the unknown input distribution matrix and $C$ the output distribution matrix.

$$
\begin{aligned}
A_{k} & :=\left[\begin{array}{cc}
1+d t \frac{7 \theta_{1} m_{2 e}^{-\frac{3}{4}}}{4} & -d t \theta_{2} \\
d t \theta_{3} & 1
\end{array}\right], B:=\left[\begin{array}{ll}
d t \theta_{2} & 0
\end{array}\right]^{T}, \\
E & :=\left[\begin{array}{ll}
0 & -d t \theta_{3}
\end{array}\right]^{T}, C:=\left[\begin{array}{ll}
0 & 1
\end{array}\right]
\end{aligned}
$$

In order to annihilate the actuator fault effect on the system, an extended unknown input observer (EUIO) is used to estimate the fault magnitude (see [16]). A control law has then to compensate the fault and be computed such that the faulty system is as close as possible to the nominal one.
We use an unknown input reconstruction method based on sliding mode observers to rewrite the system under a second form only depending on known inputs for control purposes. The system is linearized around a steady state equilibrium, the nominal state to reach, the matrix $A_{k}$ is then constant in time. We then obtain a system with new distribution matrices $A_{c}$ and $B_{c}$ under the form:

$$
\left\{\begin{array}{c}
X_{k+1}=A_{c} X_{k}+B_{c} U_{k}+B_{c} f_{k} \\
Y_{k+1}=C X_{k+1}
\end{array}\right.
$$

\section{B. Results}

With the help of the simulation software Carins (CNES), an obstruction at the input of the ferrules part has been simulated by computing a closure profile of the actuator. The closure profile is computed as a modification of the cross-sectional area of the actuator. The fault is the same for the two following examples. The first result intends to compensate the fault. We fixed the saturated value at $U_{\text {sat }}=3.59 \cdot 10^{6} \mathrm{~Pa}$ in this case.

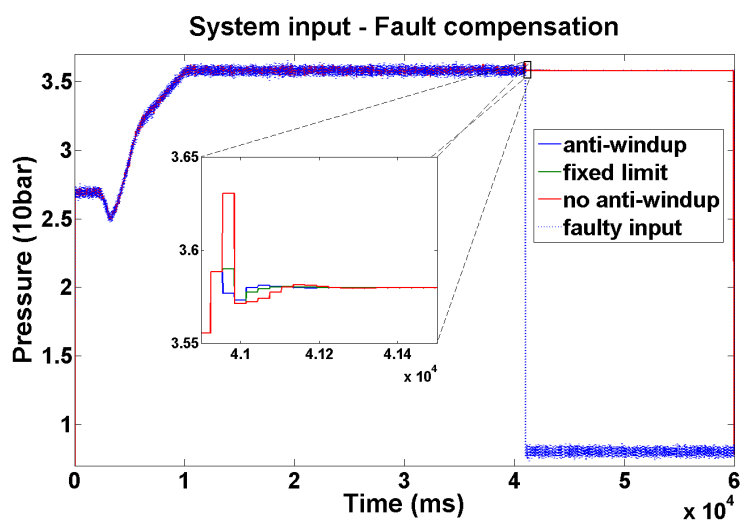

Fig. 1. Fault compensation - Control law \& system input
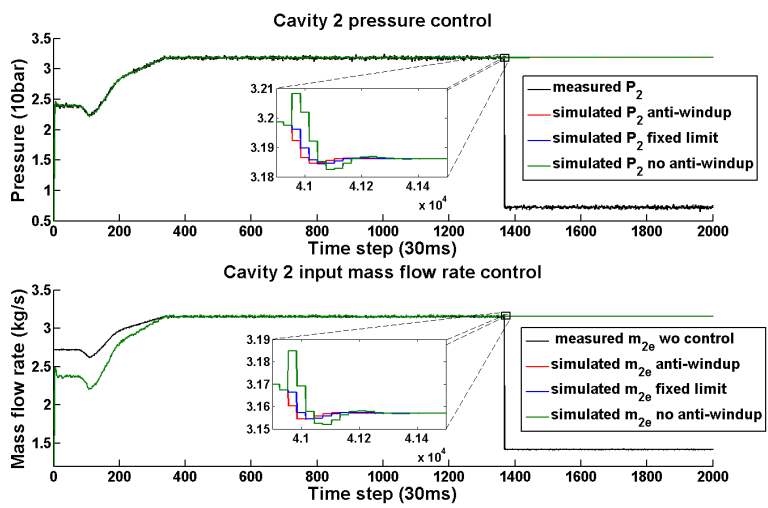

Fig. 2. Fault compensation - System state

The second result aims at compensating the fault and at converging to a different state reference than the nominal one (chosen arbitrarily). We fixed the saturated value at $U_{\text {sat }}=$ $3.782 \cdot 10^{6} \mathrm{~Pa}$ in this case (the saturation value has been 
changed in order to allow the convergence to the new state reference).

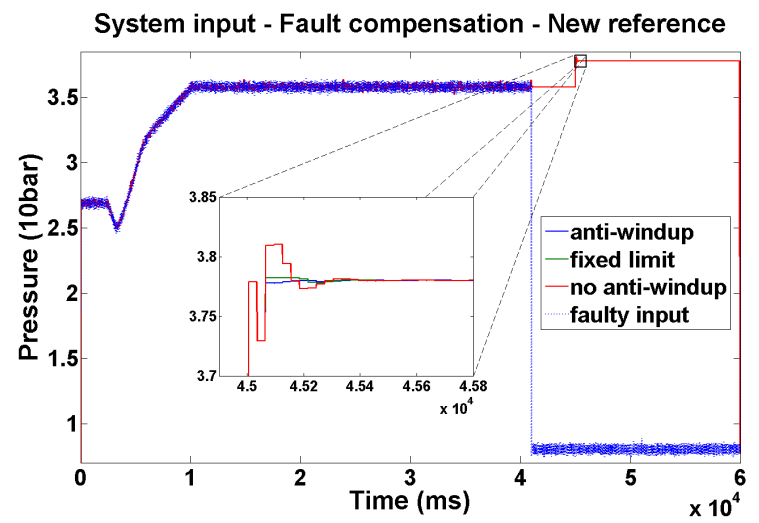

Fig. 3. Fault compensation \& reconfiguration - Control law \& system input

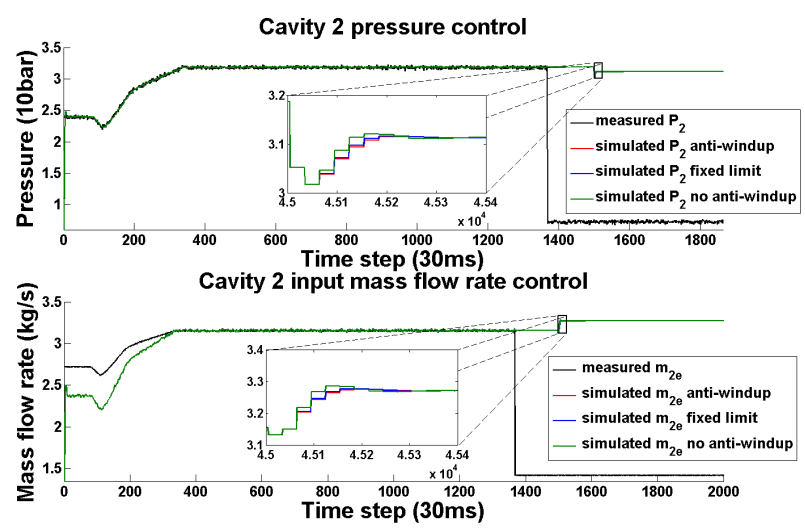

Fig. 4. Fault compensation \& reconfiguration - System state

With the first trial (Figures 1 and 2) we can see that the fault is well compensated and the convergence to the nominal value is faster. With the second trial, we can see that since the reference state dynamics is modified by the anti-windup scheme in order to ensure the exponentially asymptotic convergence, the trajectory is more stable than in the case of a fixed imposed limit (Figures 3 and 4). The new reference state dynamics is consistent with the established model, we can see that the dynamics relations between the state and the input are respected.

\section{CONCLUSION}

A method to design an anti-windup scheme in order to compute another steady point which may be reachable in the case where the previous nominal steady point cannot be reached because of the actuator failure and the effect of the saturation has been proposed. This anti-windup scheme is designed for discrete-time linear systems. Being able to shape the nominal behavior of the system is useful to take into account actuator saturation. This method is based on the resolution of linear matrix inequalities and ensures exponential asymptotic stability in an ellipsoidal domain for a polyhedral set of admissible initial states. This method was tested on a model proposed for the evolution of pressure and mass flow rates in the cooling circuit of a cryogenic test bench. Once the fault in the actuator has been detected by a FDI method, the designed FTCS based on a state observer and fault estimator (beyond the scope of this paper), a LQR control law and the proposed anti-windup scheme permits to compensate the failure and to converge if necessary to a chosen steady state. Future works will address the improvement of the anti-windup scheme method by taking into account costs functions depending on the reconfiguration objectives, for example, enlarging the stability domain. The extension to a nonlinear framework will also be considered to improve performances during transients.

\section{REFERENCES}

[1] J. Cieslak, D. Henry, A. Zolghadri, and P. Goupil, "Development of an active fault-tolerant flight control strategy," Journal of Guidance, Control, and Dynamics, vol. 31, no. 1, pp. 135-147, 2008.

[2] M. T. Hamayun, C. Edwards, and H. Alwi, "A fault tolerant control allocation scheme with output integral sliding modes," Automatica, vol. 49, no. 6, pp. 1830-1837, 2013.

[3] Z. Lin, A. A. Stoorvogel, and A. Saberi, "Output regulation for linear systems subject to input saturation," Automatica, vol. 32, no. 1, pp. $29-47,1996$.

[4] A. Benzaouia, F. Mesquine, A. Hmamed, and H. Aoufoussi, "Stability and control synthesis for discrete-time linear systems subject to actuator saturation by output feedback," Mathematical Problems in Engineering, vol. 2006, 2006.

[5] C. Gokcek, P. T. Kabamba, and S. M. Meerkov, "An LQR/LQG theory for systems with saturating actuators," IEEE Transactions on Automatic Control, vol. 46, no. 10, pp. 1529-1542, 2001.

[6] R. Mantri, A. Saberi, Z. Lin, and A. A. Stoorvogel, "Output regulation for linear discrete-time systems subject to input saturation," International Journal of Robust and Nonlinear Control, vol. 7, no. 11, pp. 1003-1021, 1997.

[7] A. A. Stoorvogel and A. Saberi, "Output regulation of linear plants with actuators subject to amplitude and rate constraints," International Journal of Robust and Nonlinear Control, vol. 9, no. 10, pp. 631-657, 1999.

[8] V. Venkataramanan, K. Peng, B. M. Chen, and T. H. Lee, "Discretetime composite nonlinear feedback control with an application in design of a hard disk drive servo system," IEEE Transactions on Control Systems Technology, vol. 11, no. 1, pp. 16-23, 2003.

[9] B. M. Chen, T. H. Lee, K. Peng, and V. Venkataramanan, "Composite nonlinear feedback control for linear systems with input saturation: Theory and an application," IEEE Transactions on Automatic Control, vol. 48, no. 3, pp. 427-439, 2003.

[10] Y. He, B. M. Chen, and C. Wu, "Improving transient performance in tracking control for linear multivariable discrete-time systems with input saturation," Systems \& Control Letters, vol. 56, no. 1, pp. 25-33, 2007.

[11] T. Hu and Z. Lin, Control systems with actuator saturation: analysis and design. Springer Science \& Business Media, 2001.

[12] J. G. da Silva Jr and S. Tarbouriech, "Anti-windup design with guaranteed regions of stability for discrete-time linear systems," Systems \& Control Letters, vol. 55, no. 3, pp. 184-192, 2006.

[13] D. Theilliol, C. Join, and Y. Zhang, "Actuator fault tolerant control design based on a reconfigurable reference input," International Journal of Applied Mathematics and Computer Science, vol. 18, no. 4, pp. 553-560, 2008.

[14] C. Sarotte, J. Marzat, H. Piet-Lahanier, A. Iannetti, M. Galeotta, and G. Ordonneau, "Actuator fault tolerant system for cryogenic combustion bench cooling circuit," 10th IFAC Symposium on Fault Detection, Supervision, Safety for Technical Processes, Warsaw, Poland, pp. 592599, 2018.

[15] L. Vingert, "Dossier de Définition et de réalisation de Mascotte V05," ONERA, Tech. Rep., 112006.

[16] M. Witczak, Modelling and estimation strategies for fault diagnosis of non-linear systems: from analytical to soft computing approaches. Springer Science \& Business Media, 2007, vol. 354. 